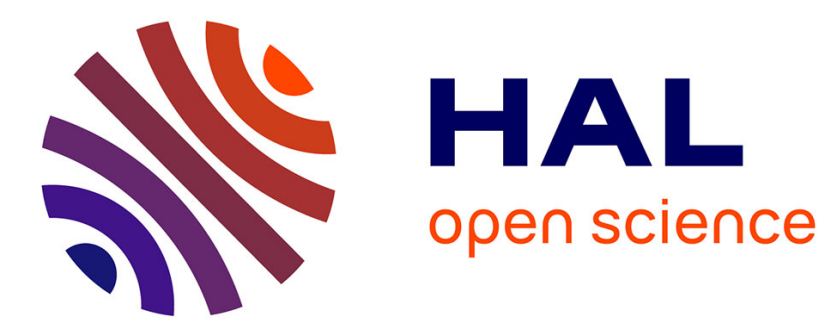

\title{
Comments on the method of harmonic balance
}

Ronald Mickens

\section{To cite this version:}

Ronald Mickens. Comments on the method of harmonic balance. Journal of Sound and Vibration, 1984, 94 (3), pp.456-460. 10.1016/S0022-460X(84)80025-5 . hal-01333728

\section{HAL Id: hal-01333728 \\ https://hal.science/hal-01333728}

Submitted on 18 Jun 2016

HAL is a multi-disciplinary open access archive for the deposit and dissemination of scientific research documents, whether they are published or not. The documents may come from teaching and research institutions in France or abroad, or from public or private research centers.
L'archive ouverte pluridisciplinaire HAL, est destinée au dépôt et à la diffusion de documents scientifiques de niveau recherche, publiés ou non, émanant des établissements d'enseignement et de recherche français ou étrangers, des laboratoires publics ou privés. 


\section{COMMENTS ON THE METHOD OF HARMONIC BALANCE}

Scientists and engineers are often confronted with a non-linear ordinary differential equation for which physical considerations suggest that one or more types of oscillatory motions are possible [1-7]. In general, except under very special conditions, exact solutions in closed form do not exist. Therefore, if analytic results on the differential equation are to be obtained, then approximation schemes must be constructed. For the case where the non-linearities are small a number of perturbation methods can be applied and approximate analytic solutions obtained [8]. However, one is often interested in looking at situations where the system is operating in a regime where the non-linear interactions are very large; consequently, perturbation techniques will not work. To my knowledge, the only technique of general applicability for obtaining approximate analytic solutions under these conditions is the method of harmonic balance [1-5]. (It should be pointed out that in the literature dealing with electrical engineering, the method goes under the name of the "describing function method"; see references $[1,2,4]$.) In general, when the method works it provides an excellent approximation to the required solution.

In spite of the fact that the method of harmonic balance has been in use for nearly 60 years, it is difficult to find in the literature a good and straightforward discussion of the advantages and limitations of the technique. The main purpose of this letter is to provide this information.

To begin; one can list the major advantages of the method of harmonic balance when it can be applied to a non-linear, ordinary differential equation to obtain an approximate analytic solution: (i) the differential equation can be of any order; the order is not restricted to be two; in general, the method is applicable to ordinary differential equations having the form

$$
L x=F,
$$

where $L$ is a linear differential operator of order $N$ and $F$ is a non-linear function of $x$ and its various derivatives; (ii) the non-linearity, represented by the function $F$, need not be "small"; (iii) limit cycle behavior and the related parameters can be easily determined; (iv) the stability of a given limit cycle can be decided in a straightforward manner; (v) most importantly, when the method works, it provides a quick and efficient procedure for obtaining good approximate analytic solutions to the non-linear differential equation of interest.

My experience in applying the method of harmonic balance to the investigation of the nature of oscillatory solutions for problems in the areas of non-linear vibrations, circuit theory, plasma physics, etc., has led me to formulate the following conditions which must be imposed on the method if it is to work [9]: (a) the non-linear function $F$ in equation (1) is assumed to be a finite sum of terms each of which has the form

$$
\left(x^{\left(n_{1}\right)}\right)^{k_{1}}\left(x^{\left(n_{2}\right)}\right)^{k_{2}} \cdots x^{l}
$$

where

$$
x^{\left(n_{1}\right)}=\mathrm{d}^{n_{1}} x / \mathrm{d} t^{n_{1}}, \quad \text { etc. }
$$

and $k_{i}$ and $l$ are non-negative integers; (b) the integers $k_{i}$ and $l$ should satisfy the relation

$$
\sum k_{i}+l=\text { odd integer; }
$$


(c) for conservative systems, where $F$ depends only on $x$, the function $F(x)$ should have only one minimum: i.e., $F(x)$ should be concave; (d) the higher harmonic terms should have small amplitudes relative to the fundamental expression.

Before proceeding, several comments are needed on (a), (b), (c) and (d) given above. First, the assumption given by (a) is merely a statement that $F$ is a polynomial function of its arguments. For almost all the non-linear differential equations which arise in practical applications, this assumption is satisfied. The condition given by (b) is a reflection of the fact that, in general, problems occur in using the method of harmonic balance when the solution to the differential equation contains a constant term [10]. The assumption (b) eliminates this difficulty. For conservative systems which have more than one stable equilibrium state, the method of harmonic balance should be applied in the neighborhood of each stable equilibrium state separately. In general, one does not expect the method to give a global description in this situation except for large values of the amplitude. The assumption given under (c) removes this problem. Finally, the method of harmonic balance is based on the assumption that the fundamental frequency term is dominant in a Fourier representation of the solution. This assumption must be checked a posteriori; (d) is merely a statement of this fact.

One is now in a position to state the required steps for obtaining an approximate analytic solution to the non-linear ordinary differential equation (1) by the method of harmonic balance. The main reason for waiting this long to give the procedure is that the discussion of the issues and assumptions under items (a)-(d) above make clear the reasons for the individual steps that are now to be presented.

(I) Check equation (1) to see whether conditions (a), (b) and (c) are satisfied.

(II) Assume a solution of the form

$$
x(t)=A \cos \omega t,
$$

substitute it into equation (1) and set the coefficient of the lowest harmonics (i.e., $\cos \omega t$ and $\sin \omega t$ ) equal to zero.

(III-A) For a conservative system, the result of performing step (II) will be a single expression

$$
h(\omega, A, \alpha)=0,
$$

where $\alpha$ denotes the fixed parameters which appear in the differential equation. This equation can then be solved to give the frequency as a function of the amplitude

$$
\omega=h_{1}(A, \alpha) \text {. }
$$

(III-B) For a non-conservative system, for which limit cycle behavior is possible, carrying out step (II) will give two expressions,

$$
h(\omega, A, \alpha)=0, \quad g(\omega, A, \alpha)=0,
$$

which can be solved to give the parameters of the possible limit cycles: i.e.,

$$
\omega_{i}=f_{1}(\alpha), \quad A_{i}=f_{2}(\alpha),
$$

where $m$ is the number of limit cycles and $i=1,2, \cdots, m$.

(IV) For a non-conservative system, determine the stability properties of all the limit cycles $[1,2]$.

(V) Check to see if the higher harmonic terms are small. In practice, this means letting $x(t)$ be represented as

$$
x(t)=A \cos \omega t+B \cos 3 \omega t
$$


carrying out steps (I), (II) and (III), and showing that

$$
|B / A| \ll 1 \text {. }
$$

(A moment's reflection should convince the reader that under conditions (a) and (b) the first harmonic to appear will be $3 \omega$. In general, even harmonics will not occur.)

(VI) Finally, for a more complete approximation to equation (1), the expression given by equation (4) can be modified to read

$$
x(t)=A \cos \omega t+B \sin \omega t .
$$

The analysis can then be carried out as described in steps (I)-(V).

The following is a small sample of the kinds of non-linear equations for which the method of harmonic balance has been applied:

$$
\begin{gathered}
\mathrm{d}^{2} x / \mathrm{d} t^{2}+x^{3}=0, \\
\mathrm{~d}^{2} x / \mathrm{d} t^{2}+\varepsilon\left[1+\beta /\left(1-x^{2}\right)\right] \mathrm{d} x / \mathrm{d} t+x=0, \\
\mathrm{~d}^{4} x / \mathrm{d} t^{4}+x+\mathrm{d}^{2}\left(x^{3}\right) / \mathrm{d} t^{2}=0 .
\end{gathered}
$$

These equations arise, respectively, in the areas of non-linear vibration [8], signal processing [7] and plasma physics [6]. To illustrate the procedure, results for equation (12), which is based on the work of Hill [11], can now be presented.

If equation (4) is substituted into equation (12) and the coefficient of the cos $\omega t$ term is set equal to zero, then the following relationship is found between the radian frequency and the amplitude:

$$
\omega(A)=\sqrt{\frac{3}{4}} A .
$$

Thus, the first approximation to the solution of equation (12) by the method of harmonic balance is

$$
x(t)=A \cos \left(\sqrt{\frac{3}{4}} \mathrm{~A}\right) \mathrm{t} .
$$

Note that the period of the motion, as determined by equation (15), is

$$
T=2 \pi / \omega=\sqrt{\frac{4}{3}}(2 \pi / A)=7 \cdot 2552 / A \text {. }
$$

For a second approximation, one assumes that the solution of equation (12) can $\mathrm{t}^{\text {- }}$ written as

$$
x(t)=A \cos \omega t+B \cos 3 \omega t .
$$

Substitution of equation (18) into equation (12) and setting the coefficients of the cos $\omega t$ and $\cos 3 \omega t$ terms equal to zero gives the following two expressions:

$$
A\left[\omega^{2}-\frac{3 A^{2}}{4}-\frac{3 A B}{4}-\frac{3 B^{2}}{2}\right]=0, \quad-9 B \omega^{2}+\frac{A^{3}}{4}+\frac{3 A^{2} B}{2}+\frac{3 B^{3}}{4}=0 .
$$

Solving equation (19a) for $\omega(A, B)$ gives

$$
\omega=\sqrt{\frac{3}{4}} A\left[1+y+2 y^{2}\right]^{1 / 2}, \quad y=B / A \text {. }
$$

If this value for $\omega$ is now substituted into equation (19b), then, with a little algebraic manipulation, one obtains

$$
51 y^{3}+27 y^{2}+21 y-1=0 .
$$

This equation is a cubic and therefore has three solutions. However, what is of interest here is seeing whether or not equation (21) has a real solution which is small: i.e., satisfies the condition given by equation (10). To proceed, assume that such a solution, $y_{s}$, exists: i.e., $\left|y_{s}\right| \ll 1$. Its approximate value can be determined by neglecting the first two terms 
in equation (21); solving the resulting equation gives

$$
y_{s} \simeq 1 / 21=0.0476 \text {. }
$$

As a check on this result, one can keep the quadratic term in equation (21) and solve this equation to obtain

$$
y_{s}^{(1)}=0.0450 \text { and } y_{s}^{(2)}=-0.8228 \text {. }
$$

Only the first root is of interest, since this is the one which is an improved approximation to the smallest root of equation (21). Thus one concludes that

$$
B / A \approx 0.045 \text {, }
$$

and, consequently, the condition of equation (10) is easily satisfied. Comparison of equations (15) and (20) shows that the radian frequency is changed by about $2 \%$.

Equation (12) has an exact solution which is [11]

$$
x(t)=A \operatorname{cn}(A t ; 1 / \sqrt{2}),
$$

where $\mathrm{cn}$ is the Jacobi elliptic cosine function. The period of this motion is

$$
T=7 \cdot 4163 / A \text {. }
$$

A Fourier expansion of equation (25) gives a ratio of the amplitudes of the $\cos 3 \omega t$ and cos $\omega t$ terms to be $0 \cdot 0450778$, as compared to the value given by equation (24). These results show clearly that the method of harmonic balance provides an excellent approximation to the solution of equation (12).

It cannot be overemphasized that the main value of the method of harmonic balance is its use in the calculation of approximate solutions for non-linear differential equations where the non-linearities are large. For situations where the non-linearities are small, various perturbation procedures should be used [8]. Furthermore, in most cases of practical interest, the method of harmonic balance need not be carried out beyond the first approximation. In general, for those non-linear differential equations for which the applicability conditions hold, the technique provides excellent estimates of the frequencyamplitude relations for conservative systems and values of the parameters for the limit cycles of non-conservative systems.

One can conclude that the harmonic balance method is a fast and efficient technique for investigating the oscillatory solutions of the class of non-linear differential equations defined by equation (1), provided that the applicability conditions (a)-(d) are satisfied.

Currently, the author is examining the use of iteration techniques to obtain approximate solutions to equation (1) where the first approximation is given by the method of harmonic balance. Such a scheme provides the possibility of calculating the higher harmonics without getting bogged down in the mass of algebraic detail which has to be dealt with in the usual application of the method of harmonic balance to this problem. Preliminary results are very encouraging.

\section{ACKNOWLEDGMENT}

The research reported in this note was supported by grants from DOE, NASA and NSF. The author thanks K. Bota and K. Oyedeji for many fruitful discussions.

Department of Physics,

R. E. Mickens

Atlanta University,

Atlanta,

Georgia 30314, U.S.A. 
1. J. C. WEST 1960 Analytical Techniques for Nonlinear Control Systems. London: English University Press.

2. D. D. SILJAK 1969 Nonlinear Systems: The Parameter Analysis and Design. New York: John Wiley and Sons.

3. I. D. HUNTLEY and R. M. JOHNSON 1983 Linear and Nonlinear Differential Equations. Chichester: Ellis Horwood Ltd.

4. D. D. WEINER and J. E. SPINA 1980 Sinusoidal Analysis and Modeling of Weakly Nonlinear Circuits. New York: Van Nostrand Reinhold Company.

5. L. A. PIPES and L. R. HARVILL 1970 Applied Mathematics for Engineers and Physicists. New York: McGraw-Hill Book Company.

6. I. V. BACHIN, V. B. KRASOVITSKII and A. M. KRYMSKII 1982 Soviet Journal of Plasma Physics 8, 336-339. Nonlinear stationary space-charge waves in beam-plasma systems.

7. S. S. Walker and J. A. Connelly 1983 Circuits Systems Signal Processes 2, 213-238. A new negative resistance oscillator model.

8. R. E. MiCKENS 1981 Nonlinear Oscillations. New York: Cambridge University Press.

9. R. E. MiCKENS 1983 Lecture Notes for Course on Computational Physics, Atlanta University.

10. A. H. NAYFEH and D. T. MOOK 1979 Nonlinear Oscillations. New York: Wiley-Interscience.

11. L. E. Hill 1983 Investigation of the Equation $\ddot{x}+x^{3}=0$. Atlanta University. 